\title{
A Path Planning Approach To Compute The Smallest Robust Forward Invariant Sets
}

\author{
Shayok Mukhopadhyay and Fumin Zhang
}

\begin{abstract}
Robustness of nonlinear systems can be analyzed by computing robust forward invariant sets (RFIS). The smallest RFIS provides the least conservative estimate of system performance under perturbations. However, computation of the smallest RFIS through brute force search can be a difficult task. We develop a novel algorithm to find the smallest RFIS for two-dimensional systems subjected to bounded additive perturbations. The algorithm leverages path planning algorithms to produce an approximation of the boundary of the smallest RFIS. The algorithm is mathematically justified, and simulation results are provided showing that the proposed algorithm can be used to find an RFIS that is very close to the smallest RFIS. The amount of computation is effectively reduced. Hence the algorithm may be generalized to higher dimensional systems with generic perturbations.
\end{abstract}

\section{INTRODUCTION}

Explicit performance bounds are desirable for robustness analysis of nonlinear systems. The methods based on input-to-state stability (ISS) may provide a conservative performance bound. Less conservative bounds on system performance may be obtained using the concept of a robust forward invariant set (RFIS) [1]. Given a system perturbed by bounded additive disturbances, it is hard to guess or establish analytically the shape or size of the smallest RFIS. Computational methods can be useful in such a situation.

Estimating the size of an RFIS is similar to the problem of estimating the region of attraction around an equilibrium point of a system. The problem of estimating the domain of attraction has been widely studied [2], [3]. Zubov's method [4] consists of solving a partial differential equation for computing the domain of attraction. In [5] the authors extend Zubov's method to perturbed dynamical systems. If Lyapunov functions can be constructed, then their sublevel sets can be used to characterize the region of attraction [6]. The sum-of-squares optimization method combined with parameter independent Lyapunov functions and branch-andbound type refinement algorithms have been applied by the authors in [7], [8]. However, the above procedure requires a family of Lyapunov functions. Lyapunov functions are not always easily found [9]. In [10], the lower and upper bounds for the size of the smallest invariant set are established for a scalar system.

This research was partially supported by ONR grants N00014-09-1-1074 and N00014-10-10712 (YIP), and NSF grants ECCS-0841195 (CAREER), CNS-0931576, OCE-1032285, and IIS-1319874. Shayok Mukhopadhyay and Fumin Zhang are with the School of Electrical and Computer Engineering, Georgia Institute of Technology, Atlanta, GA 30322, USA. The authors thank Professor Michael Malisoff from the Department of Mathematics, Louisiana State University for his helpful comments while reviewing this work. Emails:\{shayok, fumin\}@gatech.edu.
Our goal is to compute the smallest RFIS efficiently. Lyapunov based techniques are not used. This is because invariant sets computed may turn out to be the sublevel sets, hence offering conservative results. The novelty of our approach is that we leverage path planning algorithms so that the optimal path approximates the boundary of the smallest RFIS. The $A^{*}$ [11] path planning algorithm is well known to reduce the amount of computation required to find optimal paths, compared to brute force search. We derive the conditions under which the proposed algorithm terminates and produces points belonging the the boundary of the smallest RFIS. The scope of this work is limited to two dimensional systems with additive perturbations belonging to a compact set. Generalization to higher dimensions, and inclusion of more general classes of disturbances is left for future efforts.

The notation and definitions used are presented in Section II. The problem of computing the smallest RFIS is formulated in Section III followed by details of the proposed algorithm in Section IV. Mathematical justifications are provided in Section V, and Section VI presents simulation results. We present our conclusions in Section VII.

\section{Notation AND DEFinitions}

Consider the system $\dot{x}(t)=\vartheta(x(t), \delta(t))$. Here $\vartheta$ : $\mathbb{R}^{2} \times \mathbb{R}^{2} \rightarrow \mathbb{R}^{2}$, and $\delta: \mathbb{R} \rightarrow \mathbb{R}^{2}$ is a time varying bounded disturbance such that standard conditions for existence, uniqueness of solutions are met. Let $\bar{x} \in \mathbb{R}^{2}$, and $\vartheta(\bar{x}, 0)=0$. Assuming the dynamics can be written as $\vartheta(x(t), \delta(t))=f(x(t))+\delta(t)$, where $f: \mathbb{R}^{2} \rightarrow \mathbb{R}^{2}$, and the disturbance function $\delta:[0,+\infty) \rightarrow \mathcal{U}$. The set $\mathcal{U}$ is defined as $\left[-\delta_{0}, \delta_{0}\right] \times\left[-\delta_{0}, \delta_{0}\right]$, for some known value $\delta_{0} \in[0,+\infty)$. Let $\mathcal{M}_{\mathcal{U}}$ denote the set of all measurable, locally essentially bounded functions $\delta:[0,+\infty) \rightarrow \mathcal{U}$. As per [1], an RFIS is defined for a system in $\mathbb{R}^{2}$ as follows. Take any open subset $\mathcal{X}$ of $\mathbb{R}^{2}$, and any point $\mathcal{E} \in \mathcal{X}$. Consider a forward complete system $\dot{x}(t)=\mathcal{F}(x(t), \delta(t))$ with state space $\mathcal{X}$, and perturbations $\delta \in \mathcal{M}_{\mathcal{U}}$ such that standard existence and uniqueness properties of solutions are satisfied for all initial conditions in $\mathcal{X}$, and all perturbations $\delta \in \mathcal{M}_{\mathcal{U}}$. Let $\mathcal{F}(\mathcal{E}, 0)=0$. Let $\mathcal{S} \subseteq \mathcal{X}$ be any neighborhood of $\mathcal{E}$. The set $\mathcal{S}$ is an RFIS for the system $\dot{x}(t)=\mathcal{F}(x(t), \delta(t))$ with perturbations valued in $\mathcal{U}$, if all trajectories of the above system, for all initial conditions valued in $\mathcal{S}$, and perturbations $\delta \in \mathcal{M}_{\mathcal{U}}$ remain in $\mathcal{S}$ for all positive times.

Let $\mathcal{D} \subseteq \mathbb{R}^{2}$ be a region of interest, let all simple closed curves in $\mathcal{D}$ be positively oriented. Let $P, Q$ be two sequences of equal length consisting of points $p_{i}$, and 


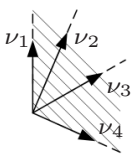

(a)

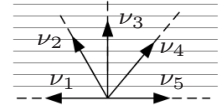

(b)
Fig. 1. Types of convex cones: (a) A pointed convex cone. (b) A convex cone which is not pointed.

$q_{j}$ respectively from Euclidean space. Define the distance between sequences $P, Q$ as $d_{2}(P, Q)=\sum_{l=1}^{n}\left\|p_{l}-q_{l}\right\|_{2}$, where $p_{l} \in P$, and $q_{l} \in Q$. Let seg $(a ; b)$ denote the segment joining any two given points $a, b$ in Euclidean space.

The following definitions are from [12]. A cone $\mathscr{C} \in \mathbb{R}^{n}$ is a set of points $x \in \mathbb{R}^{n}$ such that, if $x \in \mathscr{C}$, then for all nonnegative $\lambda \in \mathbb{R}, \lambda x \in \mathscr{C}$. A cone $\mathscr{C} \in \mathbb{R}^{n}$ is a convex cone if, and only if (a) for $x \in \mathscr{C}, \lambda x \in \mathscr{C}$ for all non-negative $\lambda \in \mathbb{R}$; (b) if $x_{1}, x_{2} \in \mathscr{C}$, then $x_{1}+x_{2} \in \mathscr{C}$. A convex cone $\mathscr{C}$ is pointed if, given any arbitrary vector $\overline{\mathbf{a}} \in \mathbb{R}^{n}$ it contains no line $\mathscr{L}=\left\{x: x=\lambda \overline{\mathbf{a}}\right.$ for all $\left.\lambda \in \mathbb{R}, x \in \mathbb{R}^{n}\right\}$. A vector $\overline{\mathbf{a}} \neq 0$ belonging to a convex cone $\mathscr{C} \in \mathbb{R}^{n}$ is an extreme ray, if $\overline{\mathbf{a}}$ cannot be expressed as a non-negative linear combination of any two linearly independent vectors from $\mathscr{C}$.

The following definitions are related to path planning on directed graphs [11]. A graph is a set $\left\{p_{i j}\right\}$ of elements called nodes and a set $\left\{\left(p_{i j}, p_{k l}\right)\right\}$ of pairs called arcs. Here $i, j, k, l$ belong to some bounded subset of $\mathbb{N}$ (natural numbers), and each arc is directed from node $p_{i j}$ to node $p_{k l}$. Given an arc $\left(p_{i j}, p_{k l}\right)$, node $p_{k l}$ is a successor node of node $p_{i j}$, the nodes $p_{i j}, p_{k l}$ are connected, and $p_{k l}$ is accessible from $p_{i j}$. The cost of an arc $\left(p_{i j}, p_{k l}\right)$ is represented by the scalar $c\left(p_{i j}, p_{k l}\right)$. A path is an ordered set of nodes with each $p_{(i+1) k}$ a successor of $p_{i j}$. Every path has a cost obtained by adding the individual costs for each arc in the path. An optimal path from $p_{i j}$ to $p_{k l}$ is a path having the smallest cost over the set of all paths from $p_{i j}$ to $p_{k l}$. Some specified non-empty set $S \subset\left\{p_{i j}\right\}$ is the source set. A single specified node $p_{i j}$ belonging to a given source set $S$ is the source node. Given a graph formed by a set of nodes $\left\{p_{i j}\right\}$ and a set of $\operatorname{arcs}\left\{\left(p_{i j}, p_{k l}\right)\right\}$, a goal set $T$ is a non-empty set of nodes $T \subset\left\{p_{i j}\right\}$ accessible from some specified source node in $\left\{p_{i j}\right\}$. Let node $p_{k l}$ belong to a particular goal set. The node $p_{k l}$ is a preferred goal node of a particular source node $p_{i j}$ if

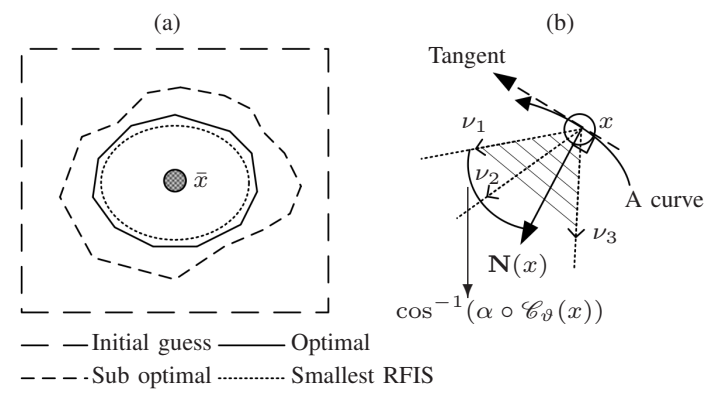

Fig. 2. Illustrations: (a) Types of solutions to the problem of finding an RFIS. (b) The quantity $\alpha \circ \mathscr{C}_{\vartheta}(x)$ for a pointed convex cone at a point $x$. the cost of an optimal path from $p_{i j}$ to $p_{k l}$ does not exceed the cost of any other path from $p_{i j}$ to any other member of the chosen goal set. In this work, the number zero is considered included in $\mathbb{N}$, and each node $p_{i j}$ has two indices $i, j$ representing the physical location of a node $p_{i j} \in \mathbb{R}^{2}$.

\section{Problem SetuP}

\section{A. Problem Statement}

Consider a system of the form $\dot{x}(t)=\vartheta(x(t), \delta(t))$ with a unique asymptotically stable equilibrium $\bar{x}$ i.e. $\vartheta(\bar{x}, 0)=0$, a particular function $\delta(t) \in \mathcal{M}_{\mathcal{U}}$, a finite constant $\delta_{0} \in$ $[0,+\infty)$, and time $t \in\left[0, T_{\max }\right]$, where $T_{\max } \in \mathbb{R}, T_{\max }>$ 0 . Given an invariant set $B \subseteq \mathcal{D}$ containing the equilibrium $\bar{x}$, find the smallest set contained in $B$ which is an RFIS for time $t \in\left[0, T_{\max }\right]$. Here $T_{\max }$ is introduced, because the computational approach proposed samples a given time varying vector field $\dot{x}(t)=\vartheta(x(t), \delta(t))$ over a finite time interval. The set $B$ is introduced because the proposed algorithm begins with an initial guess of the smallest RFIS, this is represented by the set $B$. An initial guess and expected solutions to the above problem are shown in Fig. 2 (a).

\section{B. An Optimization Problem}

Suppose the vector field $\vartheta: \mathbb{R}^{2} \times \mathbb{R}^{2} \rightarrow \mathbb{R}^{2}$, and a pointed convex cone $\mathscr{C}(x)$ with vertex at point $x \in \mathbb{R}^{2}$ are given. Let $\mathscr{C}_{\vartheta}(x)$ be a pointed convex cone with vertex at point $x \in \mathbb{R}^{2}$ such that, for all $\nu \in \mathscr{C}_{\vartheta}(x), \nu \in \operatorname{Image}(\vartheta(x, \cdot))$. Let a vector $\mathbf{N}(x)$ be given at the point $x$. The quantity $\alpha$ for a given pointed convex cone $\mathscr{C}_{\vartheta}(x)$, at point $x \in \mathbb{R}^{2}$ is written as $\alpha \circ \mathscr{C}_{\vartheta}(x)$, and defined as

$\alpha \circ \mathscr{C}_{\vartheta}(x)=\min _{\nu \in \mathscr{C}_{\vartheta}(x)}\left\{\frac{\langle\nu, \mathbf{N}(x)\rangle}{\|\nu\|\|\mathbf{N}(x)\|} \mid\|\nu\|,\|\mathbf{N}(x)\| \neq 0\right\}$

where $\nu$ is an extreme ray of $\mathscr{C}_{\vartheta}(x)$ and $\cos ^{-1}(\alpha) \in[-\pi, \pi]$. An illustration of the above definition is provided in Fig. 2 (b), where the cosine of the angle between vector $\nu_{1}, N(x)$ is the least compared with the other extreme ray (vector) $\nu_{3}$. The idea is that vector $\mathbf{N}(x)$ is a normal vector to some curve at a given point $x$. One such curve is the boundary of the smallest RFIS. The problem of finding the smallest RFIS is now the following, find all $x \in \mathcal{D}$ satisfying

$$
\min _{x \in \mathcal{D}} \alpha \circ \mathscr{C}_{\vartheta}(x) \text {, s.t. } \alpha \circ \mathscr{C}_{\vartheta}(x) \geq 0 .
$$

Solutions to the optimization problem in (2) may be obtained by a brute force search, but this is inefficient.

The following transforms the constrained optimization problem in (2) into an equivalent unconstrained one. Pick a sufficiently large positive constant $\widetilde{g}$ satisfying conditions required for Lemma 5.1 to hold, and consider the function $\eta:[-1,1] \rightarrow(0,1] \cup \widetilde{g}$ defined as below.

$$
\eta(x)=\left\{\begin{array}{lr}
x, & x \in(0,1] \\
\widetilde{g}, & x \in[-1,0]
\end{array}\right.
$$

Now, the problem in (2) is reformulated as,

$$
\min _{x \in \mathcal{D}}\left(\eta \circ \alpha \circ \mathscr{C}_{\vartheta}\right)(x) .
$$


Assigning infeasible solutions a high cost $\widetilde{g}$ using the function $\eta(\cdot)$ helps recast the problem in (4) into a path planning problem. Since the smallest RFIS around an equilibrium point $\bar{x}$ is desired, the problem in (4) is modified to include a cost related to the size of the solution set as follows

$$
\min _{x \in \mathcal{D}}\left(\eta \circ \alpha \circ \mathscr{C}_{\vartheta}\right)(x)+\lambda_{1}\|x-\bar{x}\|_{2} .
$$

Here $\lambda_{1}>0$ is a real weight which is fixed to be a desired number. Higher values for $\lambda_{1}$ penalize solutions $x$ which are farther from the equilibrium $\bar{x}$.

\section{A Path Planning Problem}

The ideal path will go around the equilibrium forming a positively oriented simple closed curve which approximates the boundary of an RFIS. Pick a convex set $B$ with a simple closed curve as its boundary such that the equilibrium $\bar{x}$ belongs to the interior of $B$. It is assumed that such a choice of a set $B$ is possible. This set $B$ is an initial guess for the desired RFIS, therefore as mentioned in section III-A, the set $B$ must itself be invariant. Sublevel sets of strict Lyapunov functions serve as good choices for $B$, thus techniques from [9] may be helpful to initialize our algorithm.

1) Discretization of the search space: The first step is to discretize the space into a radial grid. Select $N+1$ distinct points $b_{0}, b_{1}, \cdots, b_{N}$ from the boundary of $B$. Connect the equilibrium point $\bar{x}$ to each of these points. Let the index $i \in\{0,1, \cdots, N\}$. For simplicity we can choose $b_{i}$ so that the angle between each segment seg $\left(b_{i} ; \bar{x}\right)$ and the $\mathrm{x}$-axis is $\frac{2 \pi i}{N+1} \in[0,2 \pi]$. The points $b_{0}, b_{1}, b_{2}$, and $b_{N}$ are indicated on the boundary of a square set in Fig. 3 (a). The set $B$ is convex by choice, and $\bar{x}$ is in the interior of $B$. By construction, $B$ contains all line segments joining $\bar{x}$ to each point $b_{i}$. Next, discretize each segment seg $\left(b_{i} ; \bar{x}\right)$ into $n+1$ points $p_{i j}$ where $i \in\{0,1, \cdots, N\}$ and $j \in\{0,1, \cdots, n\}$ where $p_{i j}=b_{i}+j\left(\bar{x}-b_{i}\right) / n$. Notice that a point $p_{i 0}=b_{i}$ (on the boundary of $B$ ), and $p_{i n}=\bar{x}$ by construction. Let the set $\mathcal{A}_{i}$ named a segment set, be the set of points $\left\{p_{i j} \in \operatorname{seg}\left(b_{i} ; \bar{x}\right)\right\}$. The segment sets $\mathcal{A}_{0}$ to $\mathcal{A}_{N}$ are shown by dashed line segments in Fig. 3. The points $p_{i j}$ belonging to these segment sets are marked by circles. Now, convert the problem in (5)

(a)

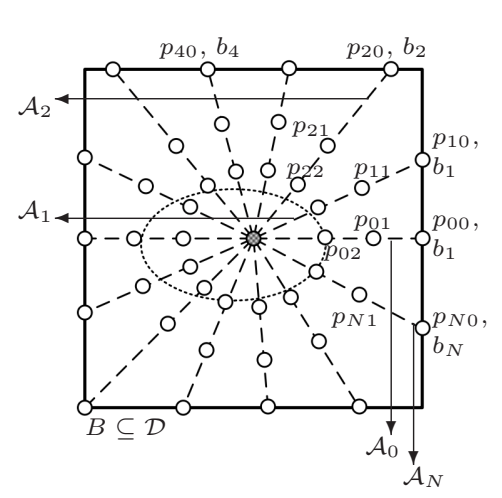

Fig. 3. (a) Discretizing the search space to find the smallest RFIS (dotted ellipse) contained within a given invariant set $\mathrm{B}$. The shaded circle is the equilibrium $\bar{x}$. (b) Embedding a directed graph structure. into the following $N+1$ separate unconstrained optimization problems. For all $i \in\{0,1, \cdots, N\}$, find an $x$ satisfying

$$
\min _{x \in \operatorname{Seg}\left(b_{i} ; \bar{x}\right)}\left(\eta \circ \alpha \circ \mathscr{C}_{\vartheta}\right)(x)+\lambda_{1}\|x-\bar{x}\|_{2}, \lambda_{1}>0 .
$$

Path planning algorithms can be used to solve (6).

2) Embedding a directed graph structure: To use path planning algorithms producing positively oriented simple closed curves around the equilibrium point as solutions to (6), the following graph structure is proposed. Excluding the equilibrium point $\bar{x}$, we connect every point in segment set $\mathcal{A}_{i}$ to every point in segment set $\mathcal{A}_{i+1}$. We do not connect any point in a segment set $\mathcal{A}_{i}$ to any other point on the same segment set, or to any point in segment set $\mathcal{A}_{i-1}$, i.e. paths cannot go backward (or clockwise around the equilibrium $\bar{x})$. To produce closed paths, the index $i$ is made to wrap around i.e. $i+1=0$ if $i=N$, and $i-1=N$ if $i=0$. This connects points (excluding the equilibrium) in segment set $\mathcal{A}_{N}$ to points in segment set $\mathcal{A}_{0}$. This graph structure, which is named $G^{\prime}$, is seen in Fig. 3 (b). Paths exist from each of the points $p_{00}, p_{01}, p_{02} \in \mathcal{A}_{0}$ directed to each of the points $p_{10}, p_{11}, p_{12} \in \mathcal{A}_{1}$. Similarly, given a point $p_{N 2} \in \mathcal{A}_{N}$, directed paths exist to points $p_{00}, p_{01}, p_{02} \in \mathcal{A}_{0}$. Now path planning is used on the graph $G^{\prime}$ to get solutions for (6).

\section{Path Planning Algorithm}

\section{A. Setting Up Sources, Goals, And Cost Functions}

The $A^{*}$ path planning algorithm requires a source node, a goal set and an evaluation function to estimate the cost of a path planned. Finding the smallest RFIS requires the source set and the goal set to be the same, i.e. segment set $\mathcal{A}_{0}$. The $A^{*}$ algorithm [11] terminates if the source node belongs to the goal set. To ensure source nodes do not belong to the goal set, a path forming a boundary of the smallest RFIS must be planned in at least two halves. The first half originating at a source node belonging to the set $\mathcal{A}_{0}$ and terminating at a preferred goal node on the segment set $\mathcal{A}_{N^{\prime}}$, where $N^{\prime}=\lfloor N / 2\rfloor$. The second half originates at the node where the first half of the path terminated in the set $\mathcal{A}_{N^{\prime}}$, and the goal set for the second half is the set $\mathcal{A}_{0}$.

Let $f\left(p_{i j}\right)$ be the actual cost of an optimal path constrained to go through node $p_{i j}$, from a given source node to a preferred goal node. The cost $f\left(p_{i j}\right)$ can be expressed as the sum $f\left(p_{i j}\right)=g\left(p_{i j}\right)+h\left(p_{i j}\right)$, where $g\left(p_{i j}\right)$ is the actual cost of a path from a source node to the node $p_{i j}$, and $h\left(p_{i j}\right)$ is the actual cost of an optimal path from $p_{i j}$ to a preferred goal node. An evaluation function $\widehat{f}\left(p_{i j}\right)$ is required so the proposed path planning algorithm eventually makes the estimated cost $\widehat{f}\left(p_{i j}\right)$ converge to the optimal cost $f\left(p_{i j}\right)$. To construct the cost functions, we first define

$$
\mathbf{N}\left(p_{i j}\right) \triangleq \Gamma_{\pi / 2}\left(p_{i j}-p_{(i-1) k}\right) .
$$

The symbol $\Gamma_{\pi / 2}$ in (7) represents the standard rotation matrix in $\mathbb{R}^{2}$. The vector $\mathbf{N}\left(p_{i j}\right)$ in (7) is therefore just the vector $p_{i j}-p_{(i-1) k}$ rotated counterclockwise by $90^{\circ}$. Then 
the cost that measures the angle between the extreme ray of the pointed convex cone $\mathscr{C}_{\vartheta}\left(p_{i j}\right)$ and $\mathbf{N}\left(p_{i j}\right)$ is

$$
c\left(p_{(i-1) k}, p_{i j}\right)=\left(\eta \circ \alpha \circ \mathscr{C}_{\vartheta}\right)\left(p_{i j}\right)+\lambda_{1}\left\|p_{i j}-\bar{x}\right\|_{2} .
$$

The following evaluation functions will be used for $A^{*}$.

$$
\begin{aligned}
& \widehat{f}\left(p_{i j}\right)=\widehat{g}\left(p_{i j}\right)+\widehat{h}\left(p_{i j}\right), \text { where } \widehat{h}\left(p_{i j}\right)=0 \\
& \widehat{g}\left(p_{i j}\right)=\min _{0 \leq k \leq n-1}\left(c\left(p_{(i-1) k}, p_{i j}\right)+\widehat{g}\left(p_{(i-1) k}\right)\right),
\end{aligned}
$$

where $j, k \in\{0,1, \cdots, n-1\}, i \in\{1, \cdots, N\}$, and the initial cost is zero i.e. $\widehat{g}\left(p_{0 k}\right)=0$ for all $k \in\{0,1, \ldots, n-$ $1\}$. The constant $\lambda_{1}$ in (8) is non-negative and is already chosen during formulation in (5). Since the index $i$ wraps around, the case of going from set $\mathcal{A}_{N}$ to $\mathcal{A}_{0}$ is given by $\widehat{g}\left(p_{0 j}\right)=\min _{0 \leq k \leq n-1}\left(c\left(p_{N k}, p_{0 j}\right)+\widehat{g}\left(p_{N k}\right)\right)$. This $\widehat{g}\left(p_{0 j}\right)$ should not be confused with the zero initial cost. The zero initial cost only applies at the start of a new path being planned from a source node in segment set $\mathcal{A}_{0}$.

If the evaluation function $\widehat{f}\left(p_{i j}\right)$ is equal at multiple points $p_{i j} \in \mathcal{A}_{i}$, then the point $p_{i j}$ with the smallest index for $j$ is selected as the preferred goal node for a node $p_{(i-1) k}$ belonging to set $\mathcal{A}_{i-1}$. Such a tie-breaker rule minimizes the quantity $\left(\eta \circ \alpha \circ \mathscr{C}_{\vartheta}\right)\left(p_{i j}\right)$ as shown in Lemma 5.2.

\section{B. The Proposed Algorithm}

Algorithm 1 presents the pseudocode proposed to find the smallest RFIS. The quantity $r$ is the iteration count. The sequence $P_{0}$ is initialized with points $\left\{b_{0}, b_{1}, \cdots, b_{N}\right\}$. Algorithm 1 generates a sequence $P_{r}$ every iteration. As $r \rightarrow \infty, P_{r}$ approaches the desired boundary. The variable $\sigma$ decides when Algorithm 1 terminates, $\sigma$ is initialized at a value greater than a given small non-zero positive constant $\epsilon$. Let $\widetilde{p}_{r, 0}$ be a source node $p_{0 j} \in \mathcal{A}_{0}$. In each iteration $r$, the first half of a circular path is planned starting from point $\widetilde{p}_{r, 0}$ to the goal set $\mathcal{A}_{N^{\prime}}$, where $N^{\prime}=\lfloor N / 2\rfloor$. The points (nodes) picked by $A^{*}$ from the segment sets $\mathcal{A}_{0}, \cdots, \mathcal{A}_{N^{\prime}}$ are stored into the sequence $P_{r}$ in the order $\left\{\widetilde{p}_{r, 0}, \widetilde{p}_{r, 1}, \widetilde{p}_{r, 2}, \cdots, \widetilde{p}_{r, N^{\prime}}\right\}$. Here a point $\widetilde{p}_{r, i}$ belongs to the segment set $\mathcal{A}_{i}, 0 \leq i \leq N^{\prime}$. The second half of a circular path is planned starting from the point $\widetilde{p}_{r, N^{\prime}} \in \mathcal{A}_{N^{\prime}}$ to the goal set $\mathcal{A}_{0}$. The sequence $P_{r}$ is updated with the points picked by $A^{*}$ from the segment sets $\mathcal{A}_{N^{\prime}+1}, \cdots, \mathcal{A}_{N}$ as

$$
P_{r}=\left\{\widetilde{p}_{r, 0}, \widetilde{p}_{r, 1}, \cdots, \widetilde{p}_{r, N^{\prime}}, \widetilde{p}_{r,\left(N^{\prime}+1\right)}, \cdots, \widetilde{p}_{r,(N-1)}, \widetilde{p}_{r, N}\right\} .
$$

To start iteration $r+1$, the point picked by $A^{*}$ from segment set $\mathcal{A}_{0}$ at the end of iteration $r$ is assigned to $\widetilde{p}_{r+1,0}$. The variable $\sigma$ is updated with the distance $d_{2}\left(P_{r}, P_{r-1}\right)$. If $\sigma \leq \epsilon$, Algorithm 1 terminates. We hypothesize that under certain conditions, the simple closed curve formed by $\operatorname{seg}\left(\widetilde{p}_{r, 0} ; \widetilde{p}_{r, 1}\right)$, $\operatorname{seg}\left(\widetilde{p}_{r, 1} ; \widetilde{p}_{r, 2}\right), \cdots, \operatorname{seg}\left(\widetilde{p}_{r, N-1} ; \widetilde{p}_{r, N}\right), \operatorname{seg}\left(\widetilde{p}_{r, N} ; \widetilde{p}_{r, 0}\right)$ is the closest approximation to the boundary of the smallest RFIS.

The constants $h, \widetilde{g}, T_{\max }$ and $\widehat{f}(\cdot)$ are used by $A^{*}$ internally. Suppose during iteration $r$ of Algorithm $1 A^{*}$ is at node $p_{(i-1) k} \in \mathcal{A}_{i-1}$ of graph $G^{\prime}$. To find the least cost path, $A^{*}$ evaluates the estimated cost $c\left(p_{(i-1) k}, p_{i j}\right)$ to every node $p_{i j} \in \mathcal{A}_{i}$ to which a directed path exists from node
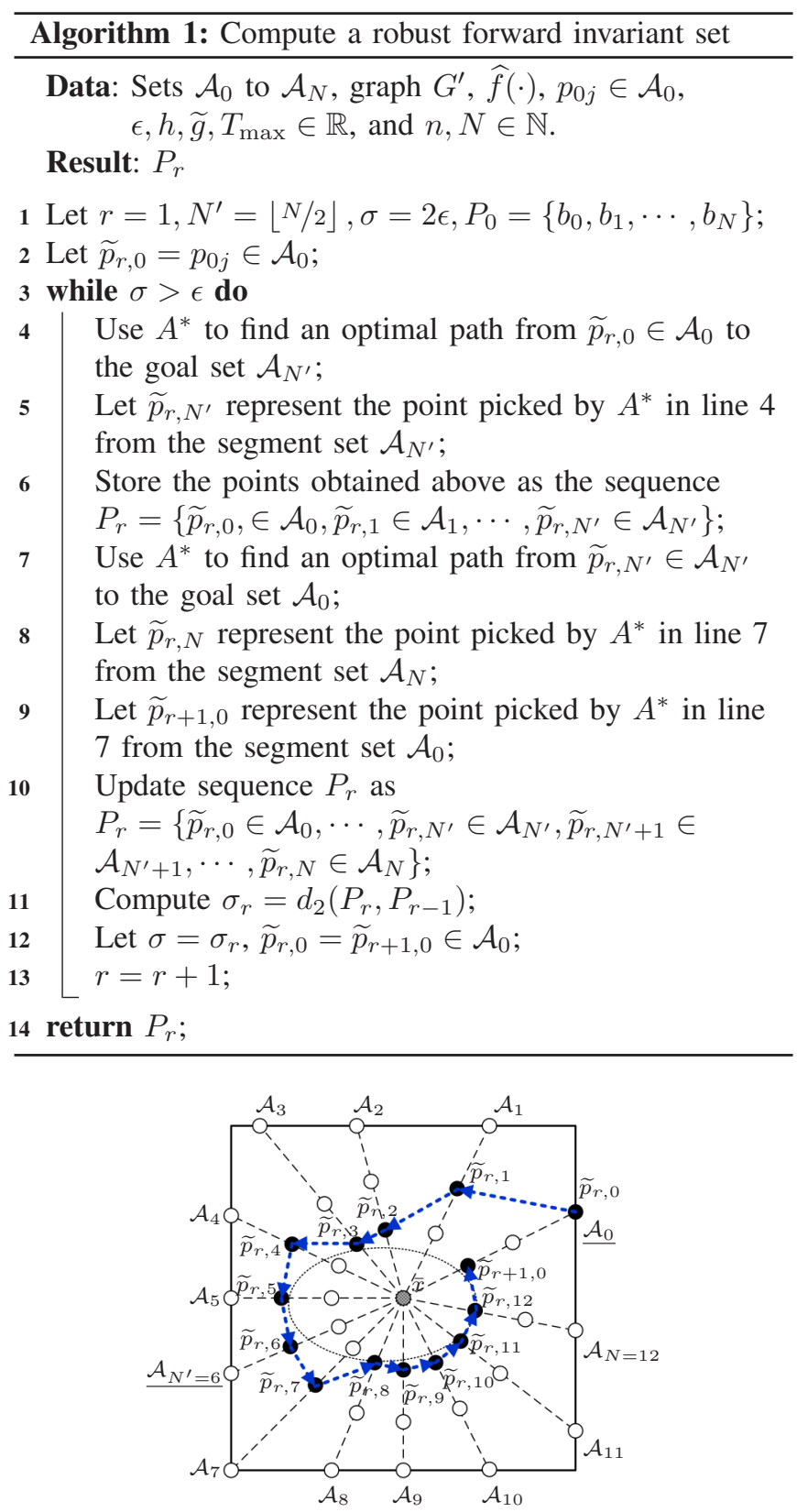

Fig. 4. An illustration of planning the required path in two halves. First from $\mathcal{A}_{0}$ to $\mathcal{A}_{N^{\prime}}$, and then from $\mathcal{A}_{N^{\prime}}$ back to $\mathcal{A}_{0}$. Set $\mathcal{A}_{0}$ is shown not lying on a horizontal line segment because any segment set can be chosen to be the set $\mathcal{A}_{0}$ as desired.

$p_{(i-1) k}$. From (8), evaluating $c\left(p_{(i-1) k}, p_{i j}\right)$ requires the evaluation of $\alpha \circ \mathscr{C}_{\vartheta}\left(p_{i j}\right)$, the details of which are as follows. As per the problem statement in section III-A, the smallest RFIS is required to be invariant for time $t \in\left[0, T_{\max }\right]$. Let $t_{l}=t_{l-1}+h$, where $l \geq 1,0 \leq t_{l} \leq T_{\max }, t_{0}=0$ and $h$ is the desired time step. At each instant $t_{l}$ find vector $\nu_{l}=\vartheta\left(p_{i j}, \delta\left(t_{l}\right)\right)$. If the set of vectors $\nu_{l}$ forms a pointed convex cone $\mathscr{C}_{\vartheta}\left(p_{i j}\right)$, then from Fig. 2 (b) it is obvious that a vector $\nu_{l}$ minimizing $\frac{\left\langle\nu_{l}, \mathbf{N}\left(p_{i j}\right)\right\rangle}{\left\|\nu_{l}\right\|\|\mathbf{N}(x)\|}$ is an extreme ray of the cone $\mathscr{C}_{\vartheta}\left(p_{i j}\right)$. The definition in (1) requires such a vector, thus the vector $\nu_{l}$ obtained as above is used to compute the quantity $\alpha \circ \mathscr{C}_{\vartheta}\left(p_{i j}\right)$. The second term in (8) is just the 
distance between the equilibrium point $\bar{x}$ and the point $p_{i j}$.

\section{MAthematical JustificAtion}

Here we justify that Algorithm 1 provides an approximation of the smallest RFIS. First, we show how an improper choice of $\widetilde{g}$ may violate constraints in (2). Let $\lambda_{1}=1$ already be fixed. Pick the positive constant $\widetilde{g}=0.1$. Let $p_{i j}, p_{i k} \in$ $\mathcal{A}_{i}$ such that $\left\|p_{i j}-\bar{x}\right\|_{2}=0.5,\left\|p_{i k}-\bar{x}\right\|_{2}=0.2$ and $\alpha \circ \mathscr{C}_{\vartheta}\left(p_{i j}\right)=0.5, \alpha \circ \mathscr{C}_{\vartheta}\left(p_{i k}\right)=-0.5$. Let $\widehat{g}\left(p_{(i-i) l}\right)=0$ for point $p_{(i-1) l} \in \mathcal{A}_{i-1}$. Suppose it is required to go from $p_{(i-1) l} \in \mathcal{A}_{i-1}$ to the segment set $\mathcal{A}_{i}$. From (3), (8) and (10), the cost to go from $p_{(i-1) l} \in \mathcal{A}_{i-1}$ to point $p_{i j} \in \mathcal{A}_{i}$ is $\widehat{f}\left(p_{i j}\right)=1$. And, the cost to go from point $p_{(i-1) l} \in \mathcal{A}_{i-1}$ to point $p_{i k} \in \mathcal{A}_{i}$ is $\widehat{f}\left(p_{i k}\right)=0.3$. If $\widetilde{g}=0.1$, then $A^{*}$ will pick point $p_{i k}$ when finding a path from a given point in set $\mathcal{A}_{i-1}$ to set $\mathcal{A}_{i}$, if costs at all other points in set $\mathcal{A}_{i}$ are greater than 0.3 . This violates constraints in (2) as $\alpha \circ \mathscr{C}_{\vartheta}\left(p_{i k}\right)<0$. Lemma 5.1 specifies conditions that $\widetilde{g}$ must satisfy to avoid such a situation. The assumptions $i \in\{0,1, \cdots, N\}, j \in\{0,1, \cdots, n-1\}, p_{i j} \in \mathcal{A}_{i}$ and $h\left(p_{i j}\right)>0$ for all $i, j$ are made for all following results.

Lemma 5.1: Let a graph $G^{\prime}$, a point $p_{(i-1) k} \in \mathcal{A}_{i-1}$, and a non-zero positive constant $\lambda_{1}$ be given. Let $\widehat{g}\left(p_{(i-1) k}\right)$ be a given non-negative number. Suppose for all $i$ there exists at least one $j$ such that $\alpha \circ \mathscr{C}_{\vartheta}\left(p_{i j}\right) \in(0,1]$, where $p_{i j} \in \mathcal{A}_{i}$. If the constant $\widetilde{g}>1+\lambda_{1}\left\|p_{i j}-\bar{x}\right\|_{2}$ for all $i, j$, then $\alpha \circ \mathscr{C}_{\vartheta}\left(p_{i j^{*}}\right) \in(0,1]$ where $p_{i j^{*}} \in \mathcal{A}_{i}$ is the preferred goal node chosen by the $A^{*}$ algorithm to go from the given point $p_{(i-1) k} \in \mathcal{A}_{i-1}$ to the segment set $\mathcal{A}_{i}$.

Proof: A proof is provided by contradiction. Suppose the constant $\widetilde{g}>1+\lambda_{1}\left\|p_{i j}-\bar{x}\right\|_{2}$, but $\alpha \circ \mathscr{C}_{\vartheta}\left(p_{i j^{*}}\right) \notin(0,1]$. By the definition of $\alpha(\cdot)$ in (1), $\cos ^{-1}(\alpha) \in[-\pi, \pi]$, this implies that $\alpha(\cdot) \in[-1,1]$. Since $\alpha \circ \mathscr{C}_{\vartheta}\left(p_{i j^{*}}\right) \notin(0,1]$ this means that $\alpha \circ \mathscr{C}_{\vartheta}\left(p_{i j^{*}}\right) \in[-1,0]$. Pick a point $p_{i j^{\prime}} \in \mathcal{A}_{i}$, such that $\alpha \circ \mathscr{C}_{\vartheta}\left(p_{i j^{\prime}}\right) \in(0,1]$. Such a choice is possible due to the assumptions. A graph $G^{\prime}$ is given, therefore there exist directed paths from the given node $p_{(i-1) k} \in \mathcal{A}_{i-1}$ to each node $p_{i j^{*}}, p_{i j^{\prime}} \in \mathcal{A}_{i}$, and from (3) $\eta(\cdot)>0$. Therefore from (8), the cost of a directed path from a node $p_{(i-1) k}$ to any node $p_{i j}$ is non-zero. Since $\widehat{h}(\cdot)=0$ is chosen in this work, hence $\widehat{h}\left(p_{i j}\right)<h\left(p_{i j}\right)$ for all $i, j$ by assumption, the requirements for using $A^{*}$ are satisfied. Hence $\widehat{f}\left(p_{i j^{*}}\right) \leq \widehat{f}\left(p_{i j^{\prime}}\right)$ by [11, Theorem 1], as $p_{i j^{*}}$ is the preferred goal node, and $A^{*}$ generates an optimal path from $p_{(i-1) k} \in \mathcal{A}_{i-1}$ to the set $\mathcal{A}_{i}$. Since the points $p_{i j^{*}}, p_{i j^{\prime}} \in \mathcal{A}_{i}$ and $p_{(i-1) k} \in \mathcal{A}_{i-1}$ are particular points which are given, therefore from definitions of $\widehat{f}(\cdot), \widehat{g}(\cdot)$ in (9), (10) the following can be written, $c\left(p_{(i-1) k}, p^{*}(i j)\right) \leq c\left(p_{(i-1) k}, p_{i j^{\prime}}^{\prime}\right)$. Now $\alpha \circ \mathscr{C}_{\vartheta}\left(p_{i j^{\prime}}^{\prime}\right) \in(0,1]$, hence from (8) and from the definition of $\eta(\cdot)$ in (3), $c\left(p_{(i-1) k}, p_{i j^{\prime}}^{\prime}\right) \leq 1+\lambda_{1}\left\|p_{i j^{\prime}}^{\prime}-\bar{x}\right\|_{2}$, hence

$$
c\left(p_{(i-1) k}, p^{*}(i j)\right) \leq 1+\lambda_{1}\left\|p_{i j^{\prime}}^{\prime}-\bar{x}\right\|_{2} .
$$

Also $\alpha \circ \mathscr{C}_{\vartheta}\left(p_{i j}^{*}\right) \in[-1,0]$, hence from (8) and from the definition of $\eta(\cdot)$ in $(3), c\left(p_{(i-1) k}, p^{*}(i j)\right)=\widetilde{g}+\lambda_{1}\left\|p_{i j}^{*}-\bar{x}\right\|_{2}$. But by assumption, $\widetilde{g}>1+\lambda_{1}\left\|p_{i j}-\bar{x}\right\|_{2}$ for all $i, j$. Hence,

$$
c\left(p_{(i-1) k}, p^{*}(i j)\right)>1+\lambda_{1}\left\|p_{i j^{\prime}}^{\prime}-\bar{x}\right\|_{2} .
$$

But (13) contradicts (12), and therefore by this contradiction we have the required result.

Therefore, so long as $\widetilde{g}$ satisfies the conditions in Lemma 5.1, Algorithm 1 picks points which do not violate the constraints in (2). The following result concerns the tiebreaker rule presented at the end of section IV-A.

Lemma 5.2: Let a point $p_{(i-1) l} \in \mathcal{A}_{i-1}$ such that $\widehat{g}\left(p_{(i-1) l}\right)$ is a non-negative number, be given. Further, let a set of points $\left\{p_{i j}\right\}$ belonging to the segment set $\mathcal{A}_{i}$ such that $\widehat{f}\left(p_{i j}\right)$ is equal for each point in the set $\left\{p_{i j}\right\}$, be given. If $j^{*}$ is the smallest index $j$ such that $p_{i j^{*}} \in\left\{p_{i j}\right\}$, then point $p_{i j^{*}}$ minimizes $\left(\eta \circ \alpha \circ \mathscr{C}_{\vartheta}\right)\left(p_{i j}\right)$ on the path from the point $p_{(i-1) l} \in \mathcal{A}_{i-1}$ to the set $\mathcal{A}_{i}$.

Proof: Let $j^{*}$ be the smallest index $j$ such that $p_{i j^{*}} \in\left\{p_{i j}\right\}$. Let $k \neq j^{*}$ be any other index $j$ such that $p_{i k} \in\left\{p_{i j}\right\}$. Since $\widehat{f}\left(p_{i j}\right)$ is equal for each point in the set $\left\{p_{i j}\right\}$, therefore $\widehat{f}\left(p_{i k}\right)=\widehat{f}\left(p_{i j^{*}}\right)$. Further, since the points $p_{i j^{*}}, p_{i k} \in \mathcal{A}_{i}$ and $p_{(i-1) k} \in \mathcal{A}_{i-1}$ are particular points, therefore from definitions of $\widehat{f}(\cdot), \widehat{g}(\cdot)$ in (9), (10) we have $c\left(p_{(i-1) l}, p_{i k}\right)+\widehat{g}\left(p_{(i-1) l}\right)=c\left(p_{(i-1) l}, p_{i j^{*}}\right)+\widehat{g}\left(p_{(i-1) l}\right)$. By the definition of $c(\cdot, \cdot)$ in (8) and re-arranging terms we get, $\lambda_{1}\left\|p_{i j^{*}}-\bar{x}\right\|_{1}-\lambda_{1}\left\|p_{i k}-\bar{x}\right\|_{2}=\left(\eta \circ \alpha \circ \mathscr{C}_{\vartheta}\right)\left(p_{i k}\right)-$ $\left(\eta \circ \alpha \circ \mathscr{C}_{\vartheta}\right)\left(p_{i j^{*}}\right)$. Since $k \neq j^{*}$, and $j^{*}$ is the smallest index $j$ such that $p_{i j^{*}} \in\left\{p_{i j}\right\}$, therefore $k>j$. Thus from the construction of the segment sets, and as the graph $G^{\prime}$ does not pass through the equilibrium $\bar{x}$, we obtain $\lambda_{1}\left\|p_{i j^{*}}-\bar{x}\right\|_{2}>\lambda_{1}\left\|p_{i k}-\bar{x}\right\|_{2}>0$. Therefore from the above discussion, and as $\eta(\cdot)$ is positive by definition in (3), we have $\left(\eta \circ \alpha \circ \mathscr{C}_{\vartheta}\right)\left(p_{i k}\right)>\left(\eta \circ \alpha \circ \mathscr{C}_{\vartheta}\right)\left(p_{i j^{*}}\right)$. Since $k$ is any other index such that $p_{i k} \in\left\{p_{i j}\right\}, k \neq j^{*}$, the required result is proved.

Assuming ties occur, Lemma 5.2 guarantees that if a point $p_{i j} \in \mathcal{A}_{i}$ with the smallest index for $j$ is selected as the preferred goal node for a node $p_{(i-1) k} \in \mathcal{A}_{i-1}$, then the quantity $\left(\eta \circ \alpha \circ \mathscr{C}_{\vartheta}\right)\left(p_{i j}\right)$ is minimized. The next result concerns the termination of Algorithm 1.

Theorem 5.3: Let the iteration count $r \geq 1$, and there exist a unique simple closed path $\bar{f}$ from the set $\mathcal{A}_{0}$ to the set $\mathcal{A}_{0}$ formed by directed paths belonging to the graph $G^{\prime}$ such that $\widehat{f}(\cdot)$ is minimized. If there exists $i$ such that $\widetilde{p}_{r, i} \in \bar{f}$ where $\widetilde{p}_{r, i} \in P_{r}$ given in (11), then $d_{2}\left(P_{r+1}, P_{r+2}\right)<\epsilon$, where $\epsilon$ is a positive constant chosen by the user of Algorithm 1 .

Proof: Let there exist $i$ such that a point $\widetilde{p}_{r, i} \in P_{r}$ is on the unique optimal path $\bar{f}$. Suppose the point $\widetilde{p}_{r, i+1} \in P_{r}$ is chosen by the $A^{*}$ algorithm to go from $\widetilde{p}_{r, i} \in P_{r}$ on the set $\mathcal{A}_{i}$ to the set $\mathcal{A}_{i+1}$ as shown in Algorithm 1. From [11] we know that $A^{*}$ selects a point $\widetilde{p}_{r, i+1} \in \mathcal{A}_{i+1}$ such that the estimated total cost $\widehat{f}(\cdot)$ to go from $\widetilde{p}_{r, i} \in \mathcal{A}_{i}$ to $\widetilde{p}_{r, i+1} \in \mathcal{A}_{i+1}$ is minimized. But there exists only a single optimal path $\bar{f}$. This implies that the point $\widetilde{p}_{r, i+1} \in \bar{f}$. Similarly, each subsequent point picked by the $A^{*}$ algorithm will lie on the optimal path $\bar{f}$. Therefore let $\widetilde{p}_{r+1,0} \in \mathcal{A}_{0}$ be the point belonging to $\bar{f}$ picked by $A^{*}$ at the end of iteration $r$. Now for iteration $r+1$ Algorithm 1 uses $A^{*}$ [11] to plan a path from the point $\widetilde{p}_{r+1,0} \in \mathcal{A}_{0}$ back around to the segment set $\mathcal{A}_{0}$, and the resulting points on the path are stored as the sequence 
$P_{r+1}=\left\{\widetilde{p}_{r+1,0}, \widetilde{p}_{r+1,1}, \cdots, \widetilde{p}_{r+1, N}\right\}$. Since $A^{*}$ generates a path such that $\hat{f}(\cdot)$ is minimized, and there exists a unique optimal path $\bar{f}$, all points $\widetilde{p}_{r+1,0}, \widetilde{p}_{r+1,1}, \cdots, \widetilde{p}_{r+1, N} \in \bar{f}$. Repeating similar arguments as above for the next iteration gives, $P_{r+2}=\left\{\widetilde{p}_{r+2,0}, \widetilde{p}_{r+2,1}, \cdots, \widetilde{p}_{r+2, N}\right\}$, where $\widetilde{p}_{r+2,0}, \widetilde{p}_{r+2,1}, \cdots, \widetilde{p}_{r+2, N} \in \bar{f}$. Since there is a single optimal path, the points $\widetilde{p}_{r+1, i} \in P_{r+1}$ are identical to the points $\widetilde{p}_{r+2, i} \in P_{r+2}$. Thus $d_{2}\left(P_{r+1}, P_{r+2}\right)=0$ which is less than any non-zero positive $\epsilon$, completing the proof.

The above result implies; if the $A^{*}$ algorithm arrives at a point on the optimal path as it searches for a least cost path, and if there is only one such optimal path, then the Algorithm 1 will quickly terminate. Based on the above result, Proposition 5.4 provides conditions under which Algorithm 1 provides an approximation of the smallest RFIS. Source nodes satisfying Proposition 5.4 can shorten the time required for Algorithm 1 to terminate.

Proposition 5.4: Suppose there exists a unique simple closed path $\bar{f}$ from set $\mathcal{A}_{0}$ to set $\mathcal{A}_{0}$ formed by directed paths belonging to graph $G^{\prime}$, such that $\hat{f}(\cdot)$ is minimized. Suppose $\widetilde{g}$ satisfies Lemma 5.1. If there exists at least one segment set $\mathcal{A}_{i}$ such that a unique point $p_{i j} \in \mathcal{A}_{i}$ also belongs to $\bar{f}$, and point $p_{i j}$ is the source node for Algorithm 1. Then the points $\widetilde{p}_{r, i} \in P_{r}$ produced by Algorithm 1 lie on the boundary of the closest approximation of the smallest RFIS.

Proof: Since $p_{i j}$ belongs to the unique optimal path $\bar{f}$, and it is the source node, applying Theorem 5.3 tells us that Algorithm 1 terminates. Since $\widetilde{g}$ satisfies Lemma 5.1, the constraints in (2) are not violated by points $\widetilde{p}_{r, i} \in P_{r}$. From our formulation in (6)-(9), these points provide the optimal solution to the problem of finding the smallest RFIS. But from assumptions, only a single optimal path exists, hence the points $\widetilde{p}_{r, i} \in P_{r}$ produced by Algorithm 1 lie on the boundary of the closest approximation of the smallest RFIS.

\section{Simulation}

For simulations we use the following problem from [1].

$$
\begin{aligned}
\dot{\rho} & =-\sin (\phi) \\
\dot{\phi} & =\left(1-\frac{\rho_{0}^{2}}{\rho^{2}}\right) \cos (\phi)-\mu \sin (\phi)+\delta(t) .
\end{aligned}
$$

We set $\epsilon=0.1, T_{\max }=6.29 \mathrm{~s}$, and $\widetilde{g}=4.5036 \times 10^{15}$ so that Lemma 5.1 holds. Further, let $\rho_{0}=1, \mu=6.42$, and the disturbance used is $\delta(t)=\delta_{0} \sin (t)$ with $\delta_{0}=0.15$. Note that these values agree with those used in [1]. The values chosen for $N, n$, and $h$ are 57,100, and 0.1. Two tests were then carried out using Algorithm 1, one with $\lambda_{1}=1$, the other with $\lambda_{1}=0.9$. For both tests Algorithm 1 runs for about $47 \mathrm{~s}$, and terminates in three iterations. Results obtained are shown in Fig. 5. The square with blue circular markers is the initial guess $B$. The curve with red square markers is obtained for $\lambda_{1}=1$, and the dashed purple curve is obtained for $\lambda_{1}=0.9$. Relaxing $\lambda_{1}$ produces a slightly larger estimate. The black dotted curves, which move from the initial guess into the interior of the computed curves, show a few sample trajectories. The sets obtained are verified to be invariant at

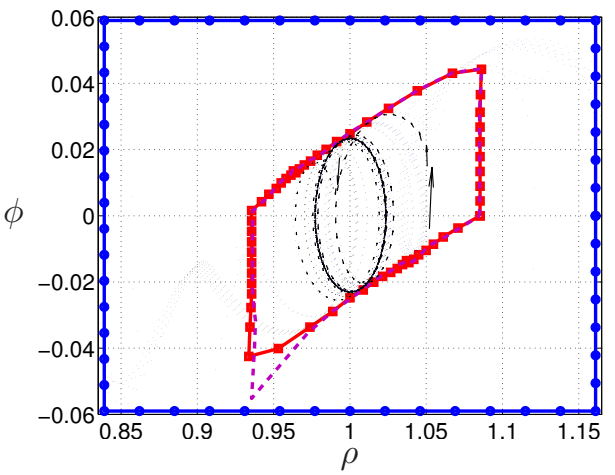

Fig. 5. The effects of using $\lambda_{1} \in(0,1]$ in Algorithm 1 with the curve tracking problem given by equations (14)-(15).

least till time $10 \times T_{\max }$. Note that the shape of the curves obtained in Fig. 5 exhibit features similar to the sets in [1].

\section{CONCLUSION}

An algorithm is presented for finding the boundary of the smallest robust forward invariant sets for two dimensional systems with bounded additive perturbations. The conditions under which the algorithm terminates and produces boundaries of an invariant set have been provided. The proposed algorithm has been applied to the curve tracking problem in [1], and the results have been verified to be invariant sets whose shape appears to be in good agreement with [1].

\section{REFERENCES}

[1] M. Malisoff, F. Mazenc, and F. Zhang, "Stability and Robustness Analysis for Curve Tracking Control using Input-to-State Stability," IEEE Transactions on Automatic Control, vol. 57, no. 5, pp. 1320$1326,2011$.

[2] A. Vannelli and M. Vidyasagar, "Maximal Lyapunov functions and domains of attraction for autonomous nonlinear systems," Automatica, vol. 21 , no. 1 , pp. 69-80, 1985.

[3] E. J. Davison and E. M. Kurak, "A computational method for determining quadratic Lyapunov functions for non-linear systems," Automatica, vol. 7, no. 5, pp. 627-636, Sep. 1971.

[4] V. I. Zubov, Methods of A.M. Lyapunov and their application. Groningen, P. Noordhoff, 1964.

[5] F. Camilli, L. Grüne, and F. Wirth, "A generalization of Zubov's method to perturbed systems," in Proceedings of the $41^{\text {st }}$ IEEE Conference on Decision and Control, vol. 3, Dec. 2002, pp. 35183523.

[6] R. Genesio, M. Tartaglia, and A. Vicino, "On the estimation of asymptotic stability regions: State of the art and new proposals," IEEE Transactions on Automatic Control, vol. 30, no. 8, pp. 747-755, Aug. 1985.

[7] V. Balakrishnan, S. Boyd, and S. Balemi, "Branch and bound algorithm for computing the minimum stability degree of parameterdependent linear systems," International Journal of Robust and Nonlinear Control, vol. 1, no. 4, pp. 295-317, 1991.

[8] U. Topcu, A. Packard, P. Seiler, and G. Balas, "Robust regionof-attraction estimation," IEEE Transactions on Automatic Control, vol. 55, no. 1, pp. 137-142, Jan. 2010.

[9] M. Malisoff and F. Mazenc, Constructions of Strict Lyapunov Functions, ser. Communications and Control Engineering. Springer, 2009.

[10] D. C. Tarraf and D. Bauso, "Robust control of networks under discrete disturbances and controls," in Proceedings of the International conference on Network Games, Control and Optimization, 2011, pp. $1-6$.

[11] P. E. Hart, N. J. Nilsson, and B. Raphael, "A formal basis for the heuristic determination of minimum cost paths," IEEE Transactions on Systems Science and Cybernetics, vol. 4, no. 2, pp. 100-107, 1968.

[12] M. J. Panik, Fundamentals of Convex Analysis. Springer, 1993. 